\title{
Coordination in Multi-agent Systems
}

\author{
Elena Nardini Andrea Omicini
}

Alma Mater Studiorum - Università di Bologna

\{elena.nardini, andrea.omicini\}@unibo.it

November 21, 2008 
(1) Environment in Multi-agent Systems

(2) Coordination in Multi-agent Systems

(3) TuCSoN

(4) Towards a Notion of Agent Coordination Context

(5) Bibliography 


\section{Outline}

(1) Environment in Multi-agent Systems

(2) Coordination in Multi-agent Systems

(3) TuCSoN

(4) Towards a Notion of Agent Coordination Context

(5) Bibliography 


\section{A Notion}

- An agent is an encapsulated computer system situated in some environment and capable of flexible, autonomous action in that environment in order to meet its design objectives [Jennings, 2001].

- A Multi-agent System (MAS) contains a number of agents that interact (cooperate, coordinate or negotiate) with one another either:

- to achieve a common objective or

- because this is necessary for them to achieve their own objectives. 


\section{The Typical Structure of MASs [Jennings, 2001]}

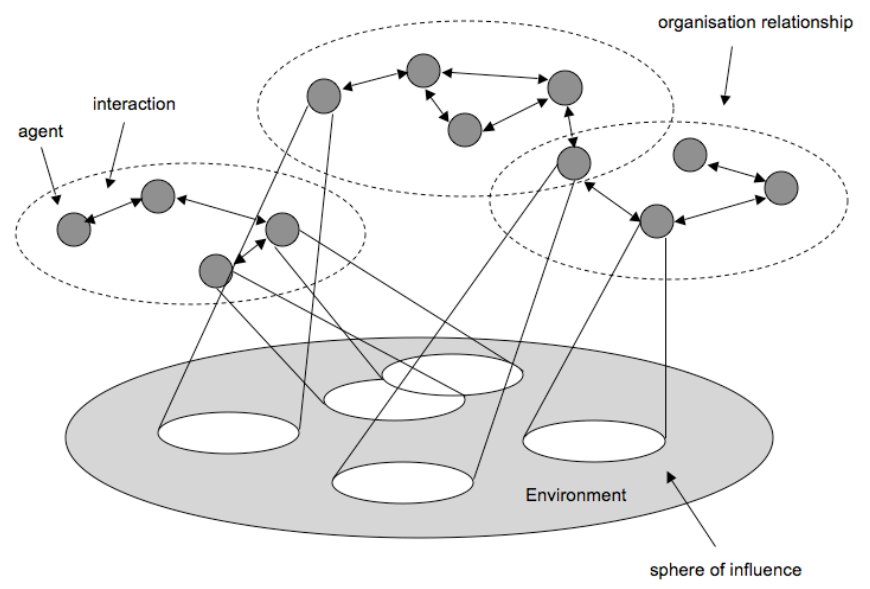




\section{Environment as a First-class Abstraction}

- The agent environment should be a first-class abstraction entity [Weyns et al., 2007].

- Structures the MAS;

- Is responsible of the activity in the MAS.

- This is even more true if we have to engineer modern software systems that are characterised by [Zambonelli and Parunak, 2003]:

- A lot of interconnected compunting systems (components);

- Situatedness;

- Openness;

- Locality in control;

- Locality in interactions.

- Ruling inter-agent and agent-environment interactions is an environment concern [Weyns et al., 2007]. 


\section{Outline}

(1) Environment in Multi-agent Systems

(2) Coordination in Multi-agent Systems

(4) Towards a Notion of Agent Coordination Context

(5) Bibliography 


\section{Coordination Meta-model}

- Coordination is the activity of managing / costraining the agent interaction space, through the adoption of first class abstractions, from design to runtime.

- These first class abstractions (coordination media), enable interaction (including communication) among agents (the coordinables), and govern it by means of suitable coordination laws [Ciancarini, 1996].

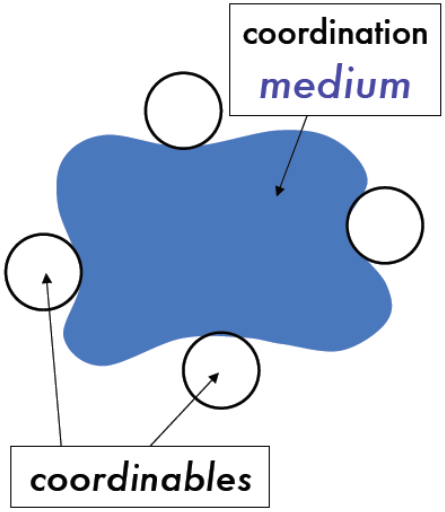




\section{Data Vs. Control Driven [Papadopoulos and Arbab, 1998]}

- Data / information-driven models

- Coordinables synchronise, cooperate, compete by accessing, consuming and producing information available in the shared data spaces.

- Control-driven models

- Coordinables synchronise, cooperate, compete by generating and reacting to signals / events in ports connected by channels.
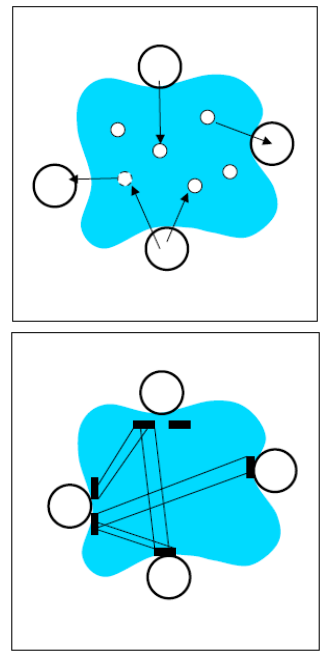


\section{Data-driven Models [Papadopoulos and Arbab, 1998]}

- Almost all coordination model belonging to this category have evolved around the notion of a shared dataspace [Roman and Cunningham, 1990].

- A shared dataspace is a common, content-addressable data structure. All processes involved in some computation can communicate among themselves only indirectly via this medium.

$\Rightarrow$ Data-driven models seems to be suitable for open applications, where a number of possibly a-priori unknown and autonomous entities have to cooperate.

$\Rightarrow$ Data-driven models better fit our application scenario than control-driven ones. 


\section{A Notable Example: Tuple Space Model}

- Coordination medium: Tuple Space.

- Multiset / bag of data object / structures called tuples.

- Communication Language: Tuples.

- Tuple $=$ ordered collection of (possibly heterogeneous) information items.

- Coordination Language: set of operations to put and retrieve associatively tuples to / from the space.

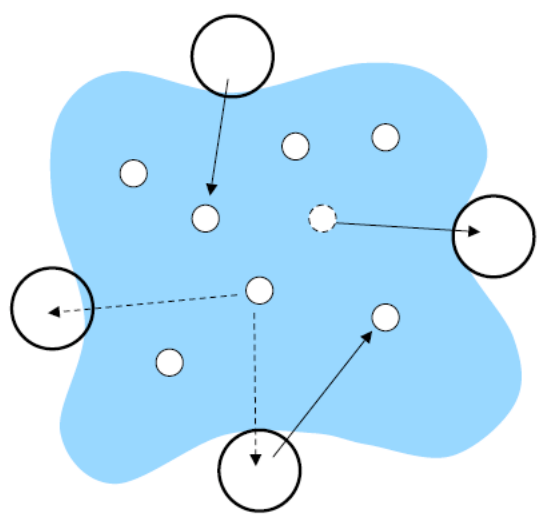




\section{Linda Coordiantion Language I [Gelernter, 1985]}

- A coordination language is the materialisation or the linguistic embodiment of a coordination model [Gelernter and Carriero, 1992].

$\Rightarrow$ It offers syntactical means with which a coordination model can be used for implementing an application.

- Linda is a coordination language for shared tuple space coordination model. 


\section{Linda Coordiantion Language II [Gelernter, 1985]}

- Communication Language

- Tuple, Templates (anti-tuples), and Tuple Matching.

- Coordination Primitives

- out $(\mathrm{T})$

$\Rightarrow$ Puts in the space the tuple T.

- in(TT)

$\Rightarrow$ Removes from the space a tuple matching the template TT.

r $\operatorname{rd}(\mathrm{TT})$

$\Rightarrow$ Reads (without removing) from the space a tuple matching the template TT. 


\section{Linda Benefits I}

- Languages Ortogonality

- Separation of coordination and computation languages that makes a program easier to be implemented and understood.

- Generality

- The same general purpose coordination language con be used in different coordination contexts, gluing different kind of computation.

$\Rightarrow$ Heterogeneity

- Gluing computation of heterogeneous computational models, all in the same coordination context.

$\Rightarrow$ Portability / Reusability

- Reusability in reusing application, implementation, tools and heterogeneous programmer expertise in the same coordination context. 


\section{Linda Benefits II}

- Generative Communication

- Until explicity withdrawn, the tuples generated by coordinables have an independent existence in the tuple space. A tuple is equally accessible to all the coordinable, but is bound to none.

$\Rightarrow$ Both senders and receivers can interact even without having prior knowledge about each others.

$\Rightarrow$ Space uncoupling.

$\Rightarrow$ Time uncoupling.

- Associative Access

- Accessing tuples through content, not through address. 


\section{Towards An Hybrid Approach}

- Linda-like coordination lacks the flexibility typical of direct and control-driven coordination models.

$\Rightarrow$ Both agent-to-agent coordination and access to local data are bound by the built-in-data-access mechanisms integrated in the shared dataspace.

- Any coordination policy not directly supported by the model has typically to be charged on agents, which are forced to implement in their code the coordination protocol required.

$\Rightarrow$ It increases the agent complexity.

$\Rightarrow$ It makes the coordination rules distributed between shared dataspaces and the agents.

$\Rightarrow$ It breakes the logical separation between coordination and algorithmic issues. 


\section{Outline}

(1) Environment in Multi-agent Systems

(2) Coordination in Multi-agent Systems

(3) TuCSoN

(4) Towards a Notion of Agent Coordination Context

(5) Bibliography 


\section{TuCSoN Coordination Model}

- Tuple Centre Spread over the Network

[Omicini and Zambonelli, 1999].

- It starts from the above considerations, by proposing a notion of an associative shared dataspace whose behaviour can be tailored according to the specific application needs.

- From tuple spaces to tuples centres [Omicini and Denti, 2001].

- Tuple centres are programmable tuple spaces.

$\Rightarrow$ Programmabile coordination media.

$\Rightarrow$ The coordination model it is the same.

- Tuple centres are distributed over the network, collected in nodes.

$\Rightarrow$ Distributed coordination media. 


\section{TuCSoN Coordination Space}

- Set of distributed nodes.

- Each TuCSoN node is an Internet node identified by the IP (logic) address.

- TuCSoN topology.

- Here, Internet topology.

- HiMAT [Cremonini et al., 1999]: hirerchical, dynamic, configurable topology.

\section{TuCSoN node}

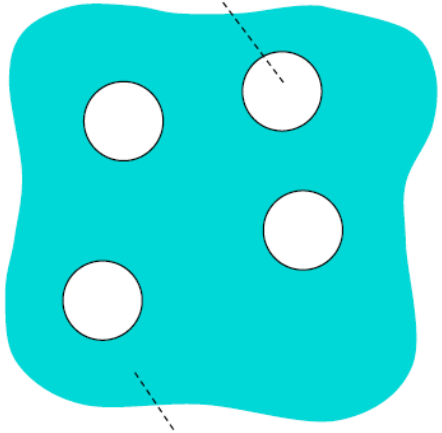

TuCSoN coordination space 


\section{TuCSoN Node / Context I}

- Each TuCSoN node defines a coordination context, providing an open / dynamic set of tuple centres as coordination media.

- Identified by means of a logic name (term).

Ex: mail (aricci), room('2.3'), ticket_dispenser, ...

- Full tuple centre identifier:

$<$ name $>$ @ $<$ node $>$.

Ex: mail (aricci) @myhome.org, room ('2.3') @ingce. unibo.it,

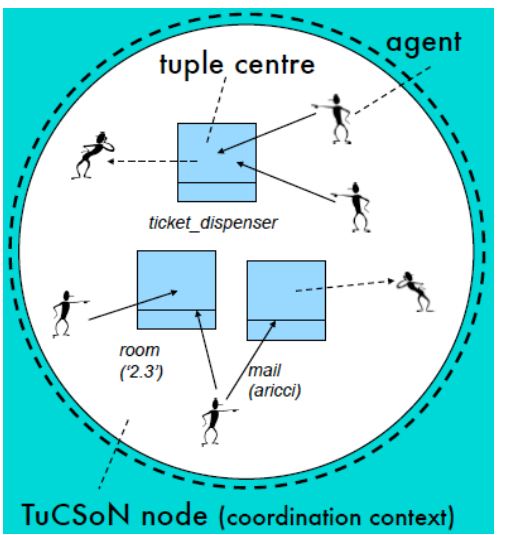
ticket_dispenser@137.204.191.188, 


\section{TuCSoN Node / Context II}

- In order to access and use the tuple centres of a node, an agent must enter the coordination context, either logically or physically (mobile agents).

- Agent Coordination Context (ACC) [Omicini, 2001].

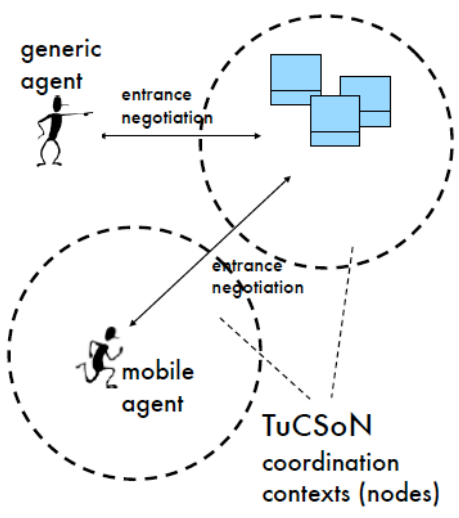




\section{Tuple Centres Features I}

- Programmable

- Tuple centre behaviour can be programmed to enact the desired coordination policies.

- ReSpecT is an example of programming language for specification of the behaviour.

$\star$ It programs as set of logic tuples (reactions, first order logic terms such as Prolog terms) specifying medium behaviour reacting to interaction events.

$\Rightarrow$ Tuple centres as a general purpose coordination media customisabled

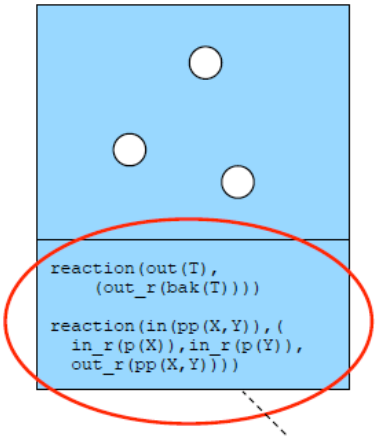

specification tuples (ReSpecT language) by means of a specification language like ReSpecT. 


\section{Tuple Centres Features II}

- Adatable at runtime

- Tuple centre behaviour can be changed / adapted dynamically, at runtime, by reprogramming the coordination media.

- Locality / encapsulation

- Tuple centres embed coordination laws.

$\Rightarrow$ A tuple centre can be a full-fledged coordination abstraction.

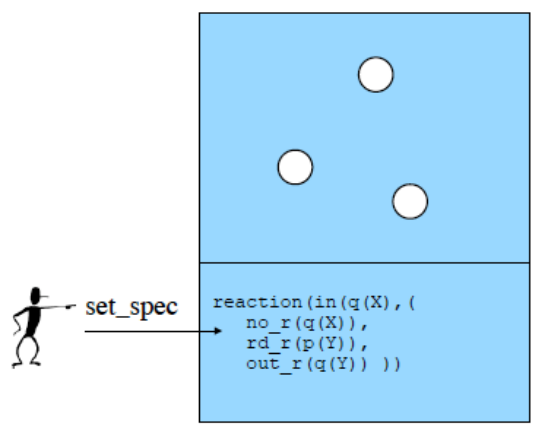

- Reaction model ensure encapsulation of low-level coordination policies. 


\section{Tuple Centres Features III}

- Inspectable at runtime

- Tuple centre behaviour can be inspected dinamically, at runtime.

- Uniformity of languages

- Same structure / primitives for communication and

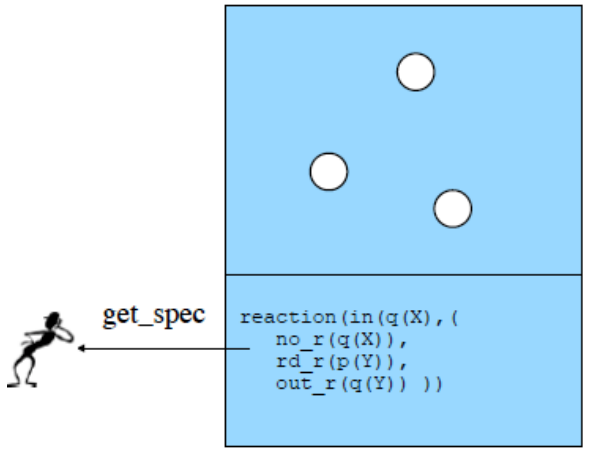
coordination 


\section{Simple Examples I}

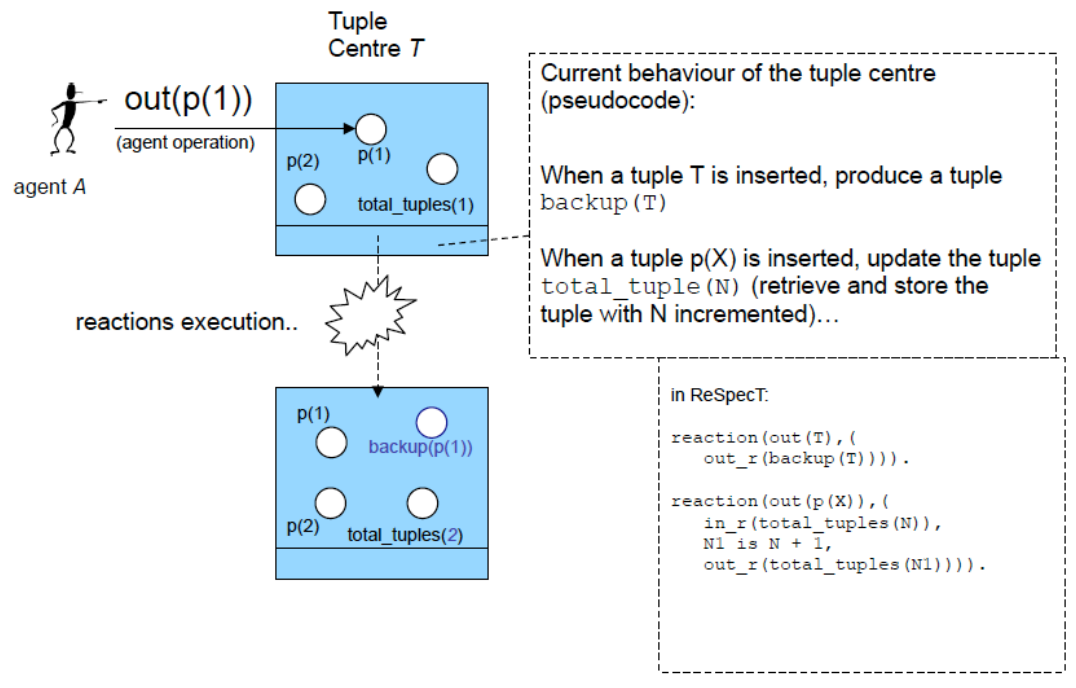




\section{Simple Examples II}

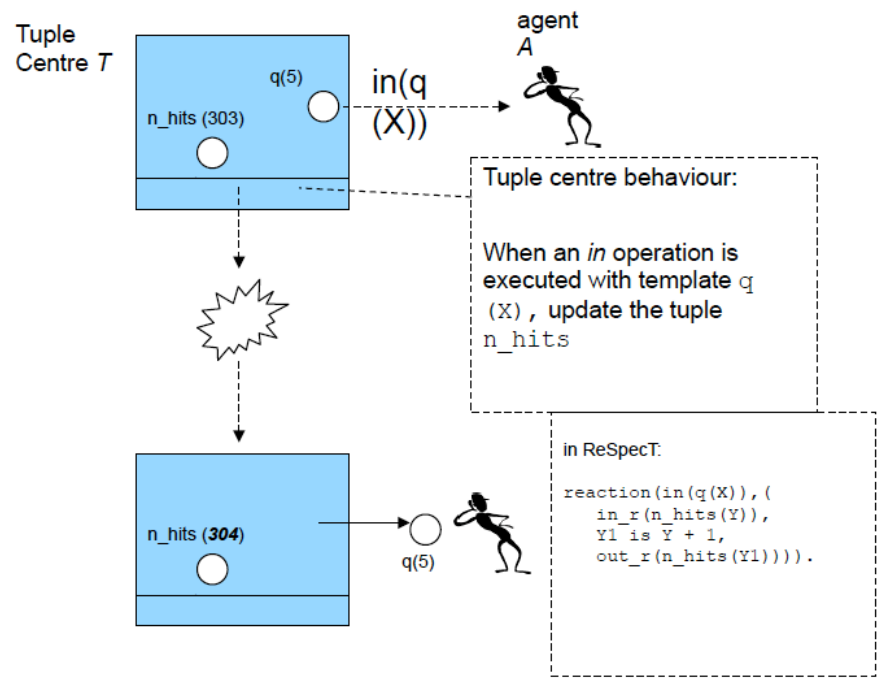




\section{TuCSoN Technology I}

- TuCSoN API

- Virtually any hosting language, currently Java and Prolog.

$\Rightarrow$ Support for Java and Prolog agents.

- Heterogeneous hardware support.

- TuCSoN Service

- Booting the TuCSoN Service daemon.

$\star$ The host becomes a TuCSoN node.

$\star$ With current version (1.4.5):

java -cp dir/tucson.jar alice.tucson.service. Node. 


\section{TuCSoN Technology II}

- TuCSoN Tools

- Inspector

$\star$ Fundamental tool to monitor tuple centre communication and coordination state, and to debug tuple centre behaviour.

$\star$ With current version (1.4.5): java -cp dir/tucson.jar alice.tucson.tools. Inspector.

- TuCSoN Shell

$\star$ Shell interface for human agents.

$\star$ With current version (1.4.5): java -cp dir/tucson.jar alice.tucson.tools. CLIAgent.

- TuCSoN technology is freely available in http://alice.unibo.it/xwiki/bin/view/TuCSoN/ 


\section{TuCSoN on the Fly}

- Booting a TuCSoN node.

- Using a tuble centre (as a human agent) by exploiting TuCSoN shell node.

- Inspecting and debugging tuple centres by exploiting TuCSoN ispector. 


\section{Development in TuCSoN}

- Building simple systems

- Experiments with the "Hello world" simple Java agent.

- Creating simple coordination among Java, human and Prolog agents. 


\section{TuCSoN in Java I}

import alice.tucson.api.*;

import alice.logictuple.*;

public class Test \{

public static void main(String[] args) throws Exception \{

TucsonContext $\mathrm{cn}$ = Tucson. enterDefaultContext();

TupleCentreId tid = new TupleCentreId("test_tc");

cn.out(tid, new LogicTuple("p", new Value("hello world")));

LogicTuple $t=c n$.in(tid, new LogicTuple("p",new $\operatorname{Var}(" \mathrm{X} "))$;

System.out.println(t);

\}

\} 


\section{TuCSoN in Java II}

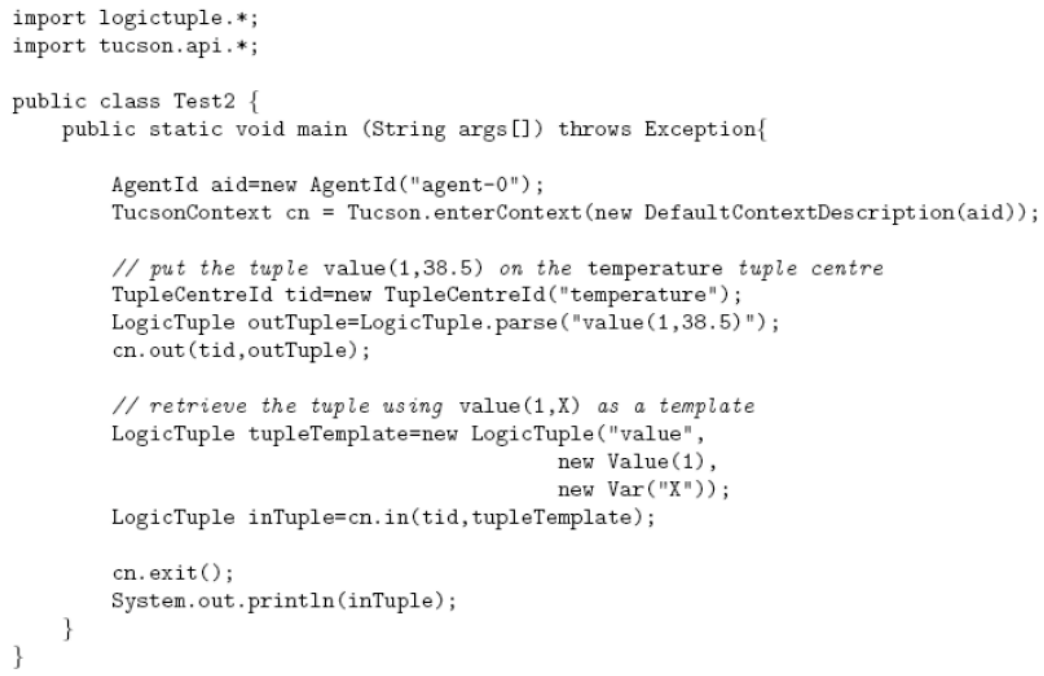




\section{TuCSoN in Java III}

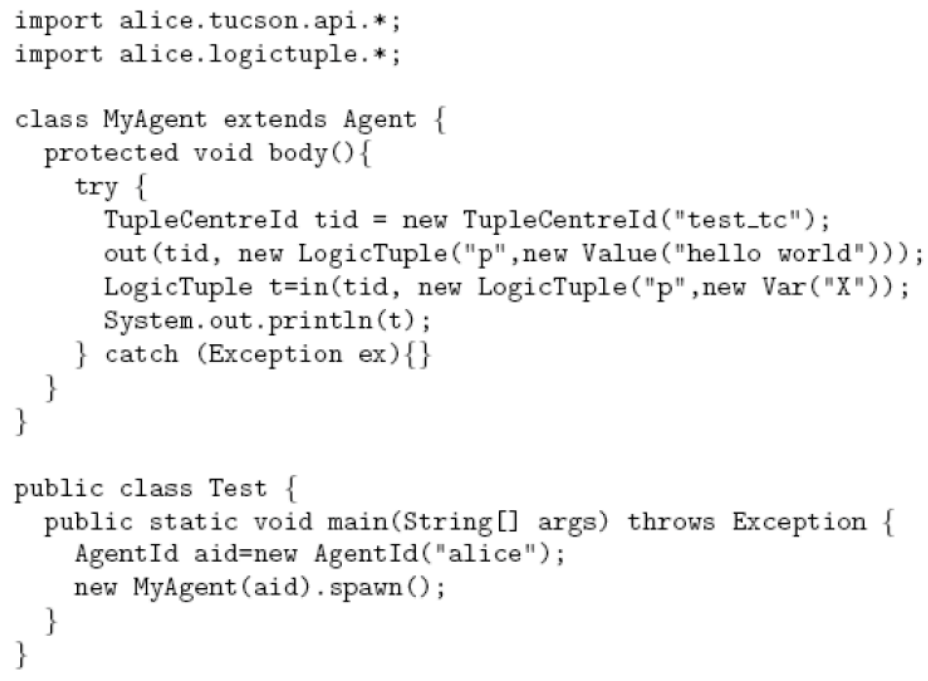




\section{TuCSoN in Prolog (tuProlog)}

:- load_library('alice.tuprologx.lib.TucsonLibrary').

:- solve(go).

go:-

test_tc ? out (p('hello world')),

test_tc? in $(\mathrm{p}(\mathrm{X}))$,

write $(\mathrm{X}), \mathrm{nl}$. 


\section{Remind}

- Ruling inter-agent and agent-environment interactions is an environment concern [Weyns et al., 2007].

$\Rightarrow \mathrm{TuCSoN}$ it is a part of agent environment.

- Until now, we have seen TuCSoN as an infrastructure supporting inter-agent interactions ...

- ... but we can also see TuCSoN as an infrastructure supporting agent-environment interactions.

- Internal environment (work environment).

- External environment (see Situated ReSpecT [Casadei and Omicini, 2009]). 


\section{Outline}

\section{(1) Environment in Multi-agent Systems}

(2) Coordination in Multi-agent Systems

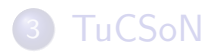

(4) Towards a Notion of Agent Coordination Context

(5) Bibliography 


\section{Coordination in Modern Software Systems}

- We call context the physical / virtual and social situation in which an agent is situated [Moran and Dourish, 2001].

$\Rightarrow$ In open world components need some form of context awareness in order to interact with both other agents and environment.

- When an agent enters in a new context, the environment should provide it with a sort of control room that provides agents with context awareness [Omicini, 2002].

- Is the only way in which the agent can perceive the environment as well as ...

- ... the only way in which the agent can interact.

$\Rightarrow$ It is possible to scale with openness of modern software systems.

$\Rightarrow$ While the environment manages social coordination, the control room manage coordination of the particular agent. 


\section{Agent Coordination Context (ACC) [Omicini, 2001] I}

- Should be works as a model for the agent environment, by describing the environment where an agent can interact.

- Subjective viewpoint: an ACC should provide agents with a suitable representation of the environment where they live, interact, and communicate.

- Objective viewpoint: an ACC should provide a framework to express the interaction within a MAS as a whole, i.e. the space of MAS interaction, that is, the admissible interactions occurring among the agents of a MAS, and between the agents of a MAS and the MAS environment. 


\section{Agent Coordination Context (ACC) [Omicini, 2001] II}

- Should enables and rules the interactions between the agent and the environment by defining the space of the admissible agent interactions.

- Subjective viewpoint: the coordination context enables in principle agents to perceive the space where they act and interact, reason about the effect of their actions and communications, and possibly affect the environment to accomplish their own goals.

- Objective viewpoint: the coordination context would allow engineers to encapsulate rules for governing applications built as agent systems, mediate the interactions amongst agents and the environment, and possibly affect them so as to change global application behaviour incrementally and dynamically. 


\section{Agent Coordination Context (ACC) [Omicini, 2001] III}

- Should be conceived not only as a tool for human designers, but also as a run-time abstraction provided as a service to agents by a suitable infrastructure.

$\Rightarrow$ agent model or behaviour is not constrained a priori.

$\Rightarrow$ Two basic stages characterize the ACC dynamics [Ricci et al., 2006]:

- ACC negotiation. An ACC is meant to be negotiated by the agents with the MAS infrastructure, in order to start a working session inside an organisation, specifying which roles to activate.

- ACC use. The agent then can use the ACC to interact with the organisation environment, by exploiting the actions / perceptions enabled by the ACC. 


\section{Agent Coordination Context (ACC) [Omicini, 2001] IV}

- Should be dynamically configurable and inspectable by both agents and humans.

- Configurability would allow a MAS to evolve at run time, by suitably adapting its behaviour to changes.

- Inspectability would allow both humans and intelligent agents to reason about the current laws of coordination as represented and embodied within coordination contexts, and to possibly change them by properly reconfigure coordination contexts according to new application needs. 


\section{ACC in MAS Infrastructure [Ricci et al., 2006] I}

- ACC framework is orthogonal both to the specific computational model(s) adopted to define agent behaviour, and to the interaction model(s) adopted to specify how agents communicate, and more generally, interact within the environment.

$\Rightarrow$ It is possible to extend any MAS infrastructure with the ACC framework in order to support the organisation and security features.

- As minimum requirements, the infrastructure must explicitly define two different models:

- It must provide a model of interaction, expressing agent / perceptions (including eventually communication).

- It should specify a basic organisational model, at least including the notion of agent identity. 


\section{ACC in MAS Infrastructure [Ricci et al., 2006] II}

- A general model of ACC can be defined, by describing three distinct aspects characterizing the ACC concept [Ricci et al., 2006]:

- ACC Interface. It defines the set of admissible operations provided by the infrastructure for interacting with the (social and resource) environment.

- ACC Contract. it is a description of the relationships between the agent and the (organisation) environment where the agent is playing, in particular of the policy enacted by the ACC ruling agent actions and interaction protocols.

- ACC Configuration. The ACC configuration is a description of the run-time state of the ACC, concerning the evolution of ongoing interaction protocols. 


\section{ACC as a Unifying Abstraction for Organisation and Security [Ricci et al., 2006] I}

- ACC can be exploited as a unifying abstraction to face a number of otherwise heterogeneous issues in the modelling and engineering of MASs where MASs are seen as structural / social settings [Ricci et al., 2006]. In particular:

- Modelling Organisation;

- Modelling Access Control;

- Modelling the Quality of Interaction;

- Modelling Relationships between Agents and Institutions. 


\section{ACC as a Unifying Abstraction for Organisation and}

Security [Ricci et al., 2006] II

- Modelling Organisation. When engineering complex software systems by adopting agent-oriented abstractions, organisation emerges a fundamental dimension [Omicini et al., 2005]. The ACC abstraction makes it possible to explicitly model the presence of an agent in an organisational context where specific structures and rules are defined.

- Modelling Access Control. Agent autonomy and system openness are among the main features that make the engineering of security particularly challenging in the context of MASs

[Omicini and Ricci, 2004]. The governing behaviour enacted by the ACC on the agent actions makes this abstraction suitable to model forms of dynamic access control to environment resources. 


\section{ACC as a Unifying Abstraction for Organisation and}

Security [Ricci et al., 2006] III

- Modelling the Quality of Interaction. As an interface, the ACC is the conceptual framework place where non-functional properties related to the quality of the interaction / communication can be suitably modelled [Ricci and Omicini, 2002].

- Modelling Relationships between Agents and Institutions. The ACC represents a contract between the agent and the institution (organisation) that released it. 


\section{ACC as a Unifying Abstraction for Organisation, Coordination, and Security IV}

- Conceiving and representing different issues exploiting a unified abstraction have several benefits [Omicini and Ricci, 2003]. In particular:

- Conceptual economy is obviously the first benefit.

- A common framework is the most obvious way to consistently support adaption and evolution of such issues.

- There are system aspects that can be modelled and engineered in their complex articulation only by considering such issues at the same time. 


\section{Experiments in TuCSoN Infrastructure}

- In [Cremonini et al., 1999] TuCSoN was extended in order to deal with security and topology issues.

- The access control model adopted, however was unrelated from organisation specification and management.

$\Rightarrow$ In [Omicini et al., 2005] the previous approach was integrated with RBAC-like architecture, by explicitly considering access control as linked to organisation structures and rules. 


\section{Outline}

(1) Environment in Multi-agent Systems

(2) Coordination in Multi-agent Systems

(3) TuCSoN

(4) Towards a Notion of Agent Coordination Context

(5) Bibliography 


\section{Bibliography I}

Casadei, M. and Omicini, A. (2009).

Situated tuple centres in ReSpecT.

In Shin, S. Y., Ossowski, S., Menezes, R., and Viroli, M., editors, 24th

Annual ACM Symposium on Applied Computing (SAC 2009),

Honolulu, Hawai'i, USA. ACM.

畨 Ciancarini, P. (1996).

Coordination models and languages as software integrators.

ACM Comput. Surv., 28(2):300-302.

- Cremonini, M., Omicini, A., and Zambonelli, F. (1999).

Multi-agent systems on the Internet: Extending the scope of coordination towards security and topology.

In Garijo, F. J. and Boman, M., editors, Multi-Agent Systems

Engineering, volume 1647 of LNAI, pages 77-88. Springer-Verlag. 


\section{Bibliography II}

9th European Workshop on Modelling Autonomous Agents in a Multi-Agent World (MAAMAW'99), Valencia, Spain, 30 June2 July 1999. Proceedings.

圂 Gelernter, D. (1985). Generative communication in linda. ACM Trans. Program. Lang. Syst., 7(1):80-112.

圄 Gelernter, D. and Carriero, N. (1992). Coordination languages and their significance. Commun. ACM, 35(2):97-107.

圂 Jennings, N. R. (2001). An agent-based approach for building complex software systems. Communications of the ACM, 44(4):35-41. 


\section{Bibliography III}

圆 Moran, T. and Dourish, P. (2001).

Introduction to this special issue on context-aware computing. Human-Computer Interaction, 20(2-4):87-95.

圆 Omicini, A. (2001).

On the notion of agent coordination context: Preliminary notes. Al*IA Notizie, XIV(4):44-46.

Omicini, A. (2002).

Towards a notion of agent coordination context.

In Marinescu, D. C. and Lee, C., editors, Process Coordination and Ubiquitous Computing, chapter 12, pages 187-200. CRC Press, Boca Raton, FL, USA.

Omicini, A. and Denti, E. (2001). From tuple spaces to tuple centres. Science of Computer Programming, 41(3):277-294. 


\section{Bibliography IV}

Omicini, A. and Ricci, A. (2003).

Reasoning about organisation: Shaping the infrastructure. Al*IA Notizie, XVI(2):7-16.

Omicini, A. and Ricci, A. (2004).

MAS organisation within a coordination infrastructure: Experiments in TuCSoN.

In Omicini, A., Petta, P., and Pitt, J., editors, Engineering Societies in the Agents World IV, volume 3071 of LNAI, pages 200-217.

Springer-Verlag.

4th International Workshop (ESAW 2003), London, UK, 29-31 October 2003. Revised Selected and Invited Papers. 


\section{Bibliography $\mathrm{V}$}

Omicini, A., Ricci, A., and Viroli, M. (2005). RBAC for organisation and security in an agent coordination infrastructure.

Electronic Notes in Theoretical Computer Science, 128(5):65-85.

2nd International Workshop on Security Issues in Coordination Models, Languages and Systems (SecCo'04), 30 August 2004. Proceedings.

Omicini, A. and Zambonelli, F. (1999).

Coordination for Internet application development.

Autonomous Agents and Multi-Agent Systems, 2(3):251-269.

Special Issue: Coordination Mechanisms for Web Agents.

惯 Papadopoulos, G. A. and Arbab, F. (1998).

Coordination models and languages.

Technical report, Amsterdam, The Netherlands, The Netherlands. 


\section{Bibliography VI}

Ricci, A. and Omicini, A. (2002).

Agent coordination contexts: Experiments in TuCSoN.

In De Paoli, F., Manzoni, S., and Poggi, A., editors, Al*IA/TABOO

Joint Workshop "Dagli oggetti agli agenti: dall'informazione alla conoscenza" (WOA 2002), Milano, Italy. Pitagora Editrice Bologna.

Ricci, A., Viroli, M., and Omicini, A. (2006).

Agent coordination contexts in a MAS coordination infrastructure. Applied Artificial Intelligence, 20(2-4):179-202.

Special Issue: Best of "From Agent Theory to Agent Implementation (AT2AI) - 4".

- Roman, G.-C. and Cunningham, H. C. (1990).

Mixed programming metaphors in a shared dataspace model of concurrency.

IEEE Trans. Softw. Eng., 16(12):1361-1373. 


\section{Bibliography VII}

Weyns, D., Omicini, A., and Odell, J. (2007).

Environment as a first class abstraction in multiagent systems. Autonomous Agents and Multi-Agent Systems, 14(1):5-30.

Zambonelli, F. and Parunak, H. V. D. (2003). Towards a paradigm change in computer science and software engineering: A synthesis.

The Knowledge Engineering Review, 18(4):329-342. 


\title{
Coordination in Multi-agent Systems
}

\author{
Elena Nardini Andrea Omicini
}

Alma Mater Studiorum - Università di Bologna

\{elena.nardini, andrea.omicini\}@unibo.it

November 21, 2008 